\title{
The holographic dark energy in non-flat Brans-Dicke cosmology
}

\author{
M.R. Setare * \\ Department of Science, Payame Noor University. Bijar, Iran
}

\begin{abstract}
In this paper we study cosmological application of holographic dark energy density in the Brans-Dicke framework. We employ the holographic model of dark energy to obtain the equation of state for the holographic energy density in non-flat (closed) universe enclosed by the event horizon measured from the sphere of horizon named $L$. Our calculation show, taking $\Omega_{\Lambda}=0.73$ for the present time, the lower bound of $w_{\Lambda}$ is -0.9 . Therefore it is impossible to have $w_{\Lambda}$ crossing -1 . This implies that one can not generate phantom-like equation of state from a holographic dark energy model in non-flat universe in the Brans-Dicke cosmology framework. In the other hand, we suggest a correspondence between the holographic dark energy scenario in flat universe and the phantom dark energy model in framework of Brans-Dicke theory with potential.
\end{abstract}

\footnotetext{
*E-mail: rezakord@ipm.ir
} 


\section{Introduction}

The accelerated expansion that based on recent astrophysical data [1], our universe is experiencing is today's most important problem of cosmology. Missing energy density - with negative pressure - responsible for this expansion has been dubbed Dark Energy (DE). Wide range of scenarios have been proposed to explain this acceleration while most of them can not explain all the features of universe or they have so many parameters that makes them difficult to fit. The models which have been discussed widely in literature are those which consider vacuum energy (cosmological constant) [2] as DE, introduce fifth elements and dub it quintessence [3] or scenarios named phantom [4] with $w<-1$, where $w$ is parameter of state.

An approach to the problem of DE arises from holographic Principle that states that the number of degrees of freedom related directly to entropy scales with the enclosing area of the system. It was shown by 'tHooft and Susskind [5] that effective local quantum field theories greatly overcount degrees of freedom because the entropy scales extensively for an effective quantum field theory in a box of size $L$ with UV cut-off $\Lambda$. As pointed out by [6], attempting to solve this problem, Cohen et al. showed [7] that in quantum field theory, short distance cut-off $\Lambda$ is related to long distance cut-off $L$ due to the limit set by forming a black hole. In other words the total energy of the system with size $L$ should not exceed the mass of the same size black hole i.e. $L^{3} \rho_{\Lambda} \leq L M_{p}^{2}$ where $\rho_{\Lambda}$ is the quantum zero-point energy density caused by UV cutoff $\Lambda$ and $M_{P}$ denotes Planck mass ( $\left.M_{p}^{2}=1 / 8 \pi G\right)$. The largest $L$ is required to saturate this inequality. Then its holographic energy density is given by $\rho_{\Lambda}=3 c^{2} M_{p}^{2} / 8 \pi L^{2}$ in which $c$ is free dimensionless parameter and coefficient 3 is for convenience.

As an application of Holographic principle in cosmology, it was studied by [8] that consequence of excluding those degrees of freedom of the system which will never be observed by that effective field theory gives rise to IR cut-off $L$ at the future event horizon. Thus in a universe dominated by DE, the future event horizon will tend to constant of the order $H_{0}^{-1}$, i.e. the present Hubble radius. The consequences of such a cut-off could be visible at the largest observable scales and particulary in the low CMB multipoles where we deal with discrete wave numbers. Considering the power spectrum in finite universe as a consequence of holographic constraint, with different boundary conditions, and fitting it with LSS, CMB and supernova data, a cosmic duality between dark energy equation of state and power spectrum is obtained that can describe the low $l$ features extremely well.

Based on cosmological state of holographic principle, proposed by Fischler and Susskind [9], the Holographic Model of Dark Energy (HDE) has been proposed and studied widely in the literature $[10,11]$. In [12] using the type Ia supernova data, the model of HDE is constrained once when $\mathrm{c}$ is unity and another time when $\mathrm{c}$ is taken as free parameter. It is concluded that the HDE is consistent with recent observations, but future observations are needed to constrain this model more precisely. In another paper [13], the anthropic principle for HDE is discussed. It is found that, provided that the amplitude of fluctuation are variable the anthropic consideration favors the HDE over the cosmological constant.

In HDE, in order to determine the proper and well-behaved system's IR cut-off, there are some difficulties that must be studied carefully to get results adapted with experiments that claim our universe has accelerated expansion. For instance, in the model proposed 
by [10], it is discussed that considering particle horizon, $R_{p}$,

$$
R_{p}=a \int_{0}^{t} \frac{d t}{a}=a \int_{0}^{a} \frac{d a}{H a^{2}}
$$

as the IR cut-off, the HDE density reads to be

$$
\rho_{\Lambda} \propto a^{-2\left(1+\frac{1}{c}\right)}
$$

that implies $w>-1 / 3$ which does not lead to accelerated universe. Also it is shown in [14] that for the case of closed universe, it violates the holographic bound.

The problem of taking apparent horizon (Hubble horizon) - the outermost surface defined by the null rays which instantaneously are not expanding, $R_{A}=1 / H$ - as the IR cut-off in the flat universe, was discussed by Hsu [15]. According to Hsu's argument, employing Friedman equation $\rho=3 M_{P}^{2} H^{2}$ where $\rho$ is the total energy density and taking $L=H^{-1}$ we will find $\rho_{m}=3\left(1-c^{2}\right) M_{P}^{2} H^{2}$. Thus either $\rho_{m}$ and $\rho_{\Lambda}$ behave as $H^{2}$. So the DE results pressureless, since $\rho_{\Lambda}$ scales as like as matter energy density $\rho_{m}$ with the scale factor $a$ as $a^{-3}$. Also, taking apparent horizon as the IR cut-off may result the constant parameter of state $w$, which is in contradiction with recent observations implying variable $w$ [16]. In our consideration for non-flat universe, because of the small value of $\Omega_{k}$ we can consider our model as a system which departs slightly from flat space. Consequently we respect the results of flat universe so that we treat apparent horizon only as an arbitrary distance and not as the system's IR cut-off.

On the other hand taking the event horizon, $R_{h}$, where

$$
R_{h}=a \int_{t}^{\infty} \frac{d t}{a}=a \int_{a}^{\infty} \frac{d a}{H a^{2}}
$$

to be the IR cut-off, gives the results compatible with observations for flat universe.

It is fair to claim that simplicity and reasonability of HDE provides more reliable frame to investigate the problem of DE rather than other models proposed in the literature[2, 3, 4]. For instance the coincidence or "why now" problem is easily solved in some models of HDE based on this fundamental assumption that matter and holographic dark energy do not conserve separately, but the matter energy density decays into the holographic energy density [17].

Some experimental data has implied that our universe is not a perfectly flat universe and recent papers have favored the universe with spatial curvature [18]. As a matter of fact, we want to remark that although it is believed that our universe is flat, a contribution to the Friedmann equation from spatial curvature is still possible if the number of e-foldings is not very large [19]. Defining the appropriate distance, for the case of non-flat universe has another story. Some aspects of the problem has been discussed in [19, 20]. In this case, the event horizon can not be considered as the system's IR cut-off, because for instance, when the dark energy is dominated and $c=1$, where $c$ is a positive constant, $\Omega_{\Lambda}=1+\Omega_{k}$, we find $\dot{R}_{h}<0$, while we know that in this situation we must be in de Sitter space with constant EoS. To solve this problem, another distance is considered- radial size of the event horizon measured on the sphere of the horizon, denoted by $L$ - and the evolution of holographic model of dark energy in non-flat universe is investigated.

Because the holographic energy density belongs to a dynamical cosmological constant, we 
need a dynamical frame to accommodate it instead of general relativity. Therefore it is worthwhile to investigate the holographic energy density in the framework of the BransDicke theory $[22,23,24]$. Einstein's theory of gravity may not describe gravity at very high energy. The simplest alternative to general relativity is Brans-Dicke scalar-tensor theory [25]. The recent interest in scalar-tensor theories of gravity arises from inflationary cosmology, supergravity and superstring theory.

In present paper, using the holographic model of dark energy in non-flat universe, we obtain equation of state for holographic dark energy density for a Brans-Dicke universe enveloped by $L$ as the system's IR cut-off. We show that Brans-Dicke theory without scalar potential correspond to quintessencial HDE, and it is impossible to have phantom era in the theory under discussion. Then we explain how the addition of scalar potential modifies the interpretation of such model as HDE.

\section{Holographic Energy Density in Brans-Dicke Frame- work}

The action of the Brans-Dicke theory in the canonical form is given by

$$
S=\int d^{4} x \sqrt{g}\left[-\frac{1}{8 \omega} \phi^{2} R+\frac{1}{2} g^{\mu v} \partial_{\mu} \phi \partial_{\nu} \phi+L_{M}\right] .
$$

where $\phi$ is the BD scalar. The non-minimal coupling term $\phi^{2} R$ where $R$ is the Ricci scalar, replaces with the Einstein-Hilbert term $\frac{1}{G_{N}} R$ in such a way that $G_{\text {eff }}^{-1}=\frac{2 \pi}{\omega} \phi^{2}$ where $G_{\text {eff }}$ is the effective gravitational constant as long as the dynamical scalar field $\phi$ varies slowly [21]. The gravitational field equations derived from the variation of the action (4) with respect to Robertson- Walker metric is

$$
\begin{gathered}
\frac{3}{4 \omega} \phi^{2}\left(\frac{\dot{a}^{2}}{a^{2}}+\frac{k}{a^{2}}\right)-\frac{1}{2} \dot{\phi}^{2}+\frac{3}{2 \omega} \frac{\dot{a}}{a} \dot{\phi} \phi=\rho \\
\frac{-1}{4 \omega} \phi^{2}\left(2 \frac{\ddot{a}}{a}+\frac{\dot{a}^{2}}{a^{2}}+\frac{k}{a^{2}}\right)-\frac{1}{\omega} \frac{\dot{a}}{a} \dot{\phi} \phi-\frac{1}{2 \omega} \ddot{\phi} \phi-\left(\frac{1}{2}+\frac{1}{2 \omega}\right) \dot{\phi}^{2}=p \\
\ddot{\phi}+3 \frac{\dot{a}}{a} \dot{\phi}-\frac{3}{2 \omega}\left(\frac{\ddot{a}}{a}+\frac{\dot{a}^{2}}{a^{2}}+\frac{k}{a^{2}}\right) \phi=0
\end{gathered}
$$

where $k$ is the curvature parameter with $k=-1,0,1$ corresponding to open, flat, closed universes respectively and $a(t)$ is the scale factor of the universe. Here $\rho=\rho_{\Lambda}+\rho_{m}$ and $p=p_{\Lambda}+p_{m}$. Where $\rho_{\Lambda}$ and $\rho_{m}$ are respectively the holographic energy density and energy density of matter. The holographic energy density $\rho_{\Lambda}$ is chosen to be

$$
\rho_{\Lambda}=\frac{3 \phi^{2}}{4 \omega L^{2}}
$$

$L$ is defined as the following form:

$$
L=\operatorname{ar}(t),
$$

here, $a$, is scale factor and $r(t)$ can be obtained from the following relation

$$
\int_{0}^{r(t)} \frac{d r}{\sqrt{1-k r^{2}}}=\int_{t}^{\infty} \frac{d t}{a}=\frac{R_{h}}{a}
$$


$R_{h}$ is event horizon. For closed universe we have (same calculation is valid for open universe by transformation)

$$
r(t)=\frac{1}{\sqrt{k}} \sin y
$$

Here $y \equiv \sqrt{k} R_{h} / a$.

The critical energy density, $\rho_{c r}$, and the energy density of curvature, $\rho_{k}$, are given by following relations respectively:

$$
\rho_{c r}=\frac{3 \phi^{2} H^{2}}{4 \omega}, \quad \rho_{k}=\frac{3 k \phi^{2}}{2 a^{2} \omega} .
$$

Now we define the dimensionless dark energy as

$$
\Omega_{\Lambda}=\frac{\rho_{\Lambda}}{\rho_{c r}}=\frac{1}{L^{2} H^{2}}
$$

Using definition $\Omega_{\Lambda}$ and relation (12), $\dot{L}$ gets:

$$
\dot{L}=H L+\operatorname{ar}(t)=\frac{1}{\sqrt{\Omega_{\Lambda}}}-\cos y
$$

Now let us consider the dark energy dominated universe. In this case the dark energy evolves according to its conservation law

$$
\dot{\rho}_{\Lambda}+3 H\left(\rho_{\Lambda}+P_{\Lambda}\right)=0
$$

Here we assume that $\phi / \phi_{0}=\left(a / a_{0}\right)^{\alpha}$, where $\alpha=\beta \kappa, \beta=\sqrt{\frac{2}{2 \omega+3}}, \kappa=\sqrt{8 \pi G}$. Substitute this relation into equation (15) and using Eq.(8), we obtain following equation of state

$$
P_{\Lambda}=\frac{-1}{3}\left(2 \alpha+1+2 \sqrt{\Omega_{\Lambda}} \cos y\right) \rho_{\Lambda}
$$

Therefore the index of the equation of state is as

$$
w_{\Lambda}=\frac{-1}{3}\left(2 \alpha+1+2 \sqrt{\Omega_{\Lambda}} \cos y\right)
$$

Because Brans-Dicke cosmology becomes standard cosmology when $\omega \rightarrow \infty$, in this case $\alpha \rightarrow 0$, according to this result, we see that the holographic dark energy has the following index of the equation of state in non-flat standard cosmology

$$
w_{\Lambda}=\frac{-1}{3}\left(1+2 \sqrt{\Omega_{\Lambda}} \cos y\right)
$$

which is exactly the result of [26]. Then $w_{\Lambda}$ is bounded from below by

$$
w_{\Lambda}=-\frac{1}{3}\left(1+2 \sqrt{\Omega_{\Lambda}}\right)
$$

taking $\Omega_{\Lambda}=0.73$ for the present time, the lower bound of $w_{\Lambda}$ is -0.9 . Therefore it is impossible to have $w_{\Lambda}$ crossing -1 . This implies that one can not generate phantomlike equation of state from a holographic dark energy model in non-flat universe in the Brans-Dicke cosmology framework. 


\section{Scalar-Tensor Theory of Gravity with Potential}

The scalar-tensor theory of gravity with a non-zero potential in the Jordan frame is given by following action [27]

$$
S=\int d^{4} x \sqrt{g}\left[\frac{1}{2}\left(F(\Phi) R-g^{\mu \nu} \partial_{\mu} \Phi \partial_{\nu} \Phi\right)-U(\Phi)+L_{M}\left(g_{\mu \nu}\right)\right]
$$

where $L_{m}$ describes dustlike matter and $F(\Phi)>0$. For a flat FRW universe, the background equations are then $[29,30]$

$$
\begin{aligned}
3 F H^{2} & =\rho_{m}+\frac{\dot{\Phi}^{2}}{2}+U-3 H \dot{F}, \\
-2 F \dot{H} & =\rho_{m}+\dot{\Phi}^{2}+\ddot{F}-H \dot{F} .
\end{aligned}
$$

The equation for the dilaton field is as

$$
\ddot{\Phi}+3 H \dot{\Phi}+\frac{d U}{d \Phi}-3\left(\dot{H}+2 H^{2}\right) \frac{d F}{d \Phi}=0 .
$$

Similar to the previous section we take $F(\phi)=\frac{\phi^{2}}{4 \omega}$, in this case the difference between actions (20) and (4) is the potential term in (20). From Eqs. $(21,22)$, one can obtain the dark energy density and pressure respectively as following

$$
\begin{gathered}
\rho_{\Lambda}=\frac{\dot{\phi}^{2}}{2}+U-3 H \dot{F}=\frac{\dot{\phi}^{2}}{2}+U-\frac{3}{2 \omega} H \dot{\phi} \phi \\
P_{\Lambda}=-F\left(2 \dot{H}+3 H^{2}\right)=\frac{\dot{\phi}^{2}}{2}\left(1+\frac{1}{\omega}\right)-U+\frac{H}{\omega} \dot{\phi} \phi+\frac{\ddot{\phi} \phi}{2 \omega}
\end{gathered}
$$

From Eqs. $(24,25)$ one can see that, when $\omega \rightarrow \infty$, the energy density and pressure of $\mathrm{DE}$ are corresponding quantities for quintessence model, it is not strange, because the scalar-tensor theory of gravity with potential is an extended quintessence model. Using Eqs. $(24,25)$, the equation of state for DE is as

$$
w_{\Lambda}=\frac{P_{\Lambda}}{\rho_{\Lambda}}=\frac{\frac{\dot{\phi}^{2}}{2}\left(1+\frac{1}{\omega}\right)-U+\frac{H}{\omega} \dot{\phi} \phi+\frac{\ddot{\phi} \phi}{2 \omega}}{\frac{\dot{\phi}^{2}}{2}+U-\frac{3}{2 \omega} H \dot{\phi} \phi}
$$

In this case, as have shown in $[29,30]$, the scalar-tensor gravity can allow crossing of the phantom divided barrier. Now we show, how the addition of scalar potential modifies the interpretation of such model as HDE.

In the flat universe, our choice for the holographic dark energy density is

$$
\rho_{\Lambda}=\frac{3 \phi^{2}}{4 \omega R_{h}^{2}}
$$

where $R_{h}$ is the event horizon

$$
R_{h}=a \int_{t}^{\infty} \frac{d t}{a}
$$

the The dimensionless dark energy is given by

$$
\Omega_{\Lambda}=\frac{\rho_{\Lambda}}{\rho_{c r}}=\frac{1}{R_{h}^{2} H^{2}}
$$


Now we reconstruct the scalar potential (phantom potential) in light of the holographic dark energy. Again we assume that $\frac{\phi}{\phi_{0}}=\left(\frac{a}{a_{0}}\right)^{\alpha}$. According to the forms of dark energy density eq.(24), one can easily derive the scalar potential term as

$$
U=\rho_{\Lambda}+\frac{\alpha}{2}\left(\frac{3}{\omega}-\alpha\right) H^{2} \phi^{2}
$$

If we establish the correspondence between the holographic dark energy and scalar field, then using Eqs.(27, 29, 30) we have

$$
U(\phi)=\left[\frac{3}{2 \omega} \Omega_{\Lambda}+\alpha\left(\frac{3}{\omega}-\alpha\right)\right] \frac{H^{2} \phi^{2}}{2} .
$$

Also, may be one expect to obtain phantom stage in the HDE model in the framework of Brans-Dicke scalar-tensor theory with potential, if one consider an infrared cutoff as a combination of the particle and future horizon [31, 32].

\section{Conclusions}

In order to solve cosmological problems and because the lack of our knowledge, for instance to determine what could be the best candidate for DE to explain the accelerated expansion of universe, the cosmologists try to approach to best results as precise as they can by considering all the possibilities they have. It is of interest to remark that in the literature, the different scenarios of DE has never been studied via considering special similar horizon, as in [28], in the standard cosmology framework, the apparent horizon, $1 / H$, determines our universe while in [22], in the Brans-Dicke cosmology framework, the universe is enclosed by event horizon, $R_{h}$. As we discussed in introduction, for flat universe the convenient horizon looks to be $R_{h}$ while in non-flat universe we define $L$ because of the problems that arise if we consider $R_{h}$ or $R_{p}$ (these problems arise if we consider them as the system's IR cut-off). In present paper, we studied $L$, as the horizon measured from the sphere of the horizon as system's IR cut-off. Then, by Considering the holographic energy density as a dynamical cosmological constant, we have obtained the equation of state for the holographic energy density in the Brans-Dicke framework. Our calculation show, taking $\Omega_{\Lambda}=0.73$ for the present time, the lower bound of $w_{\Lambda}$ is -0.9 . Therefore it is impossible to have $w_{\Lambda}$ crossing -1 . This implies that one can not generate phantom-like equation of state from an interacting holographic dark energy model in non-flat universe in the Brans-Dicke cosmology framework. In the other hand according to the Refs. [29, 30], one can explicitly demonstrate that scalar-tensor theories of gravity can predict crossing of the phantom divided barrier. But it has been known that one has to consider scalar-tensor gravity with a non-zero potential obtain such crossing. Also in $[31,32]$ the generalized holographic dark energy model was developed that among these models there are examples which may cross phantom boundary as well as effective quintessence examples.

Similar to the models of $[31,32]$ may be one expect to obtain phantom stage in the HDE model in the framework of Brans-Dicke scalar-tensor theory, if one consider an infrared cutoff as a combination of the particle and future horizon. In the other term with the choice $L$ as the combination of future and particle horizon, the HDE can be described by a phantom scalar field in Brans-Dicke theory. 
As it was mentioned in introduction, $c$ is a positive constant in holographic model of dark energy, and $(c \geq 1)$. However, if $c<1$, the holographic dark energy model also will behave like a phantom model of DE the amazing feature of which is that the equation of state of dark energy component $w_{\Lambda}$ crosses -1 . Hence, we see, the determining of the value of $c$ is a key point to the feature of the holographic dark energy and the ultimate fate of the universe as well. However, in the recent fit studies, different groups gave different values to $c$. A direct fit of the present available SNe Ia data with this holographic model indicates that the best fit result is $c=0.21[12]$. Recently, by calculating the average equation of state of the dark energy and the angular scale of the acoustic oscillation from the BOOMERANG and WMAP data on the CMB to constrain the holographic dark energy model, the authors show that the reasonable result is $c \sim 0.7$ [33]. In the other hand, in the study of the constraints on the dark energy from the holographic connection to the small $l$ CMB suppression, an opposite result is derived, i.e. it implies the best fit result is $c=2.1$ [34].

Finally I must mention that in recent work [35] I have associated the interacting holographic dark energy in non-flat universe with a phantom scalar field. I have shown that the holographic dark energy with $c<1$ can be described by the phantom in a certain way. A correspondence between the holographic dark energy and phantom has been established, and the potential of the holographic phantom and the dynamics of the field have been reconstructed. In this regard in the section 3 of this paper we have suggested a correspondence between the holographic dark energy scenario in flat universe and the phantom dark energy model in framework of Brans-Dicke theory with potential.

\section{References}

[1] S. Perlmutter et al, Nature (London), 391, 51, (1998); Knop. R et al., Astroph. J., 598, 102 (2003); A. G. Riess et al., Astrophy. J., 607, 665(2004); H. Jassal, J. Bagla and T. Padmanabhan, Phys. Rev. D, 72, 103503 (2005).

[2] For review on cosmological constant problem: P. J. E. Peebles, B. Ratra, Rev. Mod. Phys., 75, 559-606, (2003); J. Kratochvil, A. Linde, E. V. Linder, M. Shmakova, JCAP, 0407, 001, (2004).

[3] R. R. Caldwell, R. Dave and P. J. Steinhardt, Phys. Rev. Lett., 80, (1998) 1582; I. Zlater, L. Wang and P. J. Steinhardt, Phys. Rev. Lett., 82, (1999), 896; T. Chiba, gr-qc/9903094; M. S. Turner and M. White Phys. Rev. D, 56, (1997), 4439.

[4] R. R. Caldwell, Phys. Lett. B 545, 23, (2002); R. R. Caldwell, M. Kamionkowsky and N. N. Weinberg, Phys, Rev, Lett, 91, 071301, (2003); S. Nojiri and S. D. Odintsov, Phys. Lett., B 562, (2003), 147; S. Nojiri and S. D. Odintsov, Phys. Lett., B 565, (2003), 1; S. Nojiri and S. D. Odintsov, Phys. Rev., D, 72, 023003, (2005); S. Nojiri, S. D. Odintsov, O. G. Gorbunova, J. Phys., A, 39, 6627, (2006); S. Capozziello, S. Nojiri, S. D. Odintsov, Phys. Lett. B 632, 597, (2006).

[5] L. Susskind, J. Math. Phys, 36, (1995), 6377-6396.

[6] Y. S. Myung, Phys. Lett. B 610, (2005), 18-22. 
[7] A. Cohen, D. Kaplan and A. Nelson, Phys. Rev. Lett 82, (1999), 4971.

[8] K. Enqvist, S. Hannestad and M. S. Sloth, JCAP, 0502, (2005) 004.

[9] W. Fischler and L. Susskind, hep-th/9806039.

[10] M. Li, Phy. Lett. B, 603, 1, (2004).

[11] D. N. Vollic, hep-th/0306149; H. Li, Z. K. Guo and Y. Z. Zhang, astro-ph/0602521; X. Zhang, Int. J. Mod. Phys. D 14, 1597, (2005); X. Zhang, and F. Q. Wu, Phys. Rev. D 72, 043524, (2005); Z. Chang, F. Q. Wu, and X. Zhang, Phys. Lett. B633, 14, (2006); J. P. B. Almeida and J. G. Pereira, gr-qc/0602103; D. Pavon and W. Zimdahl, hep-th/0511053; Y. Gong, Phys. Rev., D, 70, (2004), 064029; B. Wang, E. Abdalla, R. K. Su, Phys. Lett., B, 611, (2005); M. R. Setare, Phys. Lett. B641, 130, (2006); M. R. Setare, and S. Shafei, JCAP, 09, 011, (2006).

[12] Q. G. Huang, Y. Gong, JCAP, 0408, (2004),006.

[13] Q. G. Huang, M. Li, JCAP, 0503, (2005), 001.

[14] R. Easther and D. A. Lowe hep-th/9902088.

[15] S. D. H. Hsu, Phys. Lett. B, 594, 13, (2004).

[16] U. Alam, V. Sahni, T. D. Saini, A. A. Starobinsky, Mon. Not. Roy. Astron. Soc., 354, 275 (2004); D. Huterer and A. Cooray, Phys. Rev., D, 71, 023506, (2005), Y. Wang and M. Tegmark, astro-ph/0501351.

[17] B. Gumjudpai, T. Naskar, M. Sami and S. Tsujikawa, JCAP 0506, 007, (2005); E. J. Copeland, M. Sami and S. Tsujikawa, hep-th/0603057; B. Hu, Y. Ling, Phys. Rev. D73 (2006) 123510; X. Zhang, Phys. Rev. D 74, 103505, (2006);M. R. Setare, Phys. Lett. B642, 1, (2006); M. R. Setare, Phys. Lett. B642, 421, (2006).

[18] C. L. Bennet et al, astrophys. J. Suppl., 148, (2003) 1; D. N. Spergel, Astrophys. J. Suppl., 148, (2003), 175; K. Ichikawa et al, astro-ph/0605481.

[19] Q. G. Huang and M. Li, JCAP, 0408, 013, (2004).

[20] B. Guberina, R. Horvat and H. Nikolić, Phys. Rev. D 72, 125011, (2005).

[21] M. Arik, M. C. Calik, Mod. Phys. Lett. A 21, 1241, (2006).

[22] Y. Gong, Phys. Rev. D, 70, 064029, (2004).

[23] H. Kim, H. W. Lee, and Y. S. Myung, Phys. Lett. B 628, 11, (2005).

[24] D. F. Torres, Phys. Rev. D66, 043522, (2002).

[25] C. Brans and C. H. Dicke, Phys. Rev. 124, 925 (1961).

[26] Q. G. Huang, and M. Li, JCAP 0408, 013, (2004).

[27] P.G. Bergmann, Int. J. Theor. Phys. 1, 25 (1968); K. Nordtvedt, Astrophys. J. 161, 1059 (1970); R. Wagoner, Phys. Rev. D 1, 3209 (1970). 
[28] P. C. Davies, Class. Quantum. Grav. L225, 4, (1987).

[29] L. Perivolaropoulos, JCAP, 0510, 001, (2005).

[30] R. Gannouji, D. Polarski, A. Ranquet, and A. A. Starobinsky, JCAP 0609, 016, (2006).

[31] E. Elizalde, S. Nojiri, S. D. Odintsov and P. Wang, Phys. Rev. D71,103504, (2005).

[32] S. Nojiri and S. D. Odintsov, Gen. Rel. Grav 38, 1285, (2006).

[33] H. C. Kao, W. L. Lee and F. L. Lin, astro-ph/0501487.

[34] J. Shen, B. Wang, E. Abdalla and R. K. Su, hep-th/0412227.

[35] M. R. Setare "Interacting Holographic Phantom" 